\title{
An EVN survey of hard spectrum gamma ray sources
}

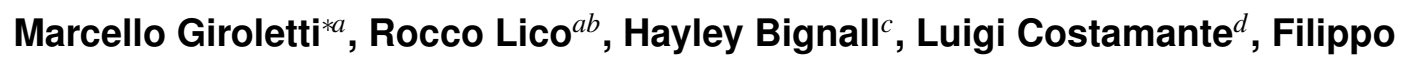
D’Ammando $^{a b}$, Gabriele Giovannini ${ }^{a b}$, Monica Orienti ${ }^{a}$, Cormac Reynolds ${ }^{c}$, Gino Tosti $^{\text {de }}$

a INAF - Istituto di Radioastronomia di Bologna, Italy

${ }^{b}$ Dipartimento di Fisica e Astronomia, Università di Bologna, Italy

${ }^{c}$ Curtin Institute for Radio Astronomy, Curtin University, Australia

${ }^{d}$ Dipartimento di Fisica, Università degli Studi di Perugia, Italy

${ }^{e}$ Istituto Nazionale di Fisica Nucleare, Sezione di Perugia, Italy

E-mail: giroletti@ira.inaf.it, rocco.lico@unibo.it,

h.bignallecurtin.edu.au, luigic2011@gmail.com,

dammando@ira.inaf.it, ggiovanneira.inaf.it, orientieira.inaf.it,

c.reynoldsecurtin.edu.au, tosti@pg.infn.it

The first Fermi-LAT catalogue of sources detected above $10 \mathrm{GeV}$ (1FHL) is an ideal sample to characterize the properties of the most extreme gamma-ray sources in the Universe. It is wide, deep, and unbiased, being selected from an all-sky survey. We present 1.6 GHz European VLBI Network (EVN) observations of the sky regions of the 71 1FHL sources in the northern sky without any existing VLBI observation, partly classified as blazars and partly unidentified. The VLBI observations help to confirm and characterize the proposed blazars (typically of the enigmatic class with high frequency synchrotron peak), and to find candidate counterparts for the unidentified sources. We also explore the presence of a flux-flux correlation between radio and $E>10$ $\mathrm{GeV}$ gamma rays: such a radio-gamma correlation was found to be highly significant for blazars detected at $E<10 \mathrm{GeV}$ but it is entirely elusive for $\mathrm{TeV}$ sources; our data fill the gap between these two conflicting extremes. For the first time, we report results revealing only a weak radiogamma-ray connection at $E>10 \mathrm{GeV}$; this lack of a strong correlation has a physical rather than observational origin.

12th European VLBI Network Symposium and Users Meeting

7-10 October 2014

Cagliari, Italy

\footnotetext{
* Speaker.
} 


\section{Introduction}

Operating on-board the Fermi satellite launched in 2008, the Large Area Telescope (LAT) has greatly enhanced our knowledge and understanding of the high energy universe. With its 2.4 sr field of view and a broad energy range (in principle, from $20 \mathrm{MeV}$ to greater than $300 \mathrm{GeV}$ ), the LAT has provided a deep, uniform sky survey ideal for population studies. Blazars, i.e. radio loud active galactic nuclei (AGN) whose emission is predominantly due to the Doppler boosted non-thermal radiation from a relativistic jet pointing near to the observer's line of sight, dominate the census of the Fermi detected sources; they make up about $50-60 \%$ of the total number of sources in all the Fermi-LAT catalogues. When focusing on just the first catalogue of AGNs detected byFermi-LAT (the so-called 1LAC, Abdo et al., 2010), Ackermann et al. (2011) have revealed a strong and highly significant correlation between the radio flux density and the gamma-ray energy flux.

At even higher energies (Very High Energies, VHE, $E>0.1 \mathrm{TeV}$ ), the study of the Universe relies upon the detection on the ground of Cherenkov radiation produced in the atmosphere by gamma-ray photons. In this energy band, the current instrumentation is dominated by small field of view instruments which operate mainly in pointed mode (MAGIC, HESS, VERITAS). Therefore, we lack a large, deep, homogeneous survey at VHE and no systematic study of the existence of a radio-VHE correlation has been made so far. At present, only 47 VHE AGNs are known, out of a total population of 151 detected sources ${ }^{1}$. Moreover, there are physical reasons to expect that such correlation might be elusive at best; these include the anti-correlation between synchrotron luminosity and spectral energy distribution (SED) peak frequency (the so-called blazar sequence, Fossati et al., 1998) and the photon-photon interaction between the VHE radiation and the extragalactic background light (EBL), which decreases the observed VHE flux for distant sources.

Therefore, the first Fermi catalog of high energy sources $(E>10 \mathrm{GeV}$, hereafter 1FHL, Ackermann et al., 2013) is at present the best resource to discuss the connection between radio and gamma rays above several GeV. The $1 \mathrm{FHL}$ is based on three years of all-sky survey by Fermi; it contains 514 sources, $76 \%$ of which are AGNs.

In the present contribution, we report on the current status of a project aimed at studying the radio-gamma ray correlation in the northern hemisphere with 1FHL and VLBI data. In Section 2, we present our datasets from gamma-ray and radio observations; in Section 3, we show some preliminary results; finally, we discuss the results and the prospects for the continuation of the project in Section 4.

\section{Observations and datasets}

\subsection{Gamma-ray observations}

We show in Figure 1 the three years sky map of the gamma-ray counts above $10 \mathrm{GeV}$ from which the 1FHL has been extracted. The 1FHL contains 514 sources with $T S>25^{2}$. This detection threshold corresponds to variable flux limits, which depend on the source photon index, the diffuse background emission, and the exposure; at high galactic latitude, it is typically in the range $(5-$

\footnotetext{
${ }^{1}$ see http: //tevcat.uchicago.edu

${ }^{2}$ The test statistics is defined as $T S=2 \times\left(\log L_{1}-\log L_{0}\right)$ with $L_{0}$ the likelihood of the null-hypothesis model as compared to the likelihood of the model to be tested, $L_{1}$; see Mattox et al. (1996)
} 


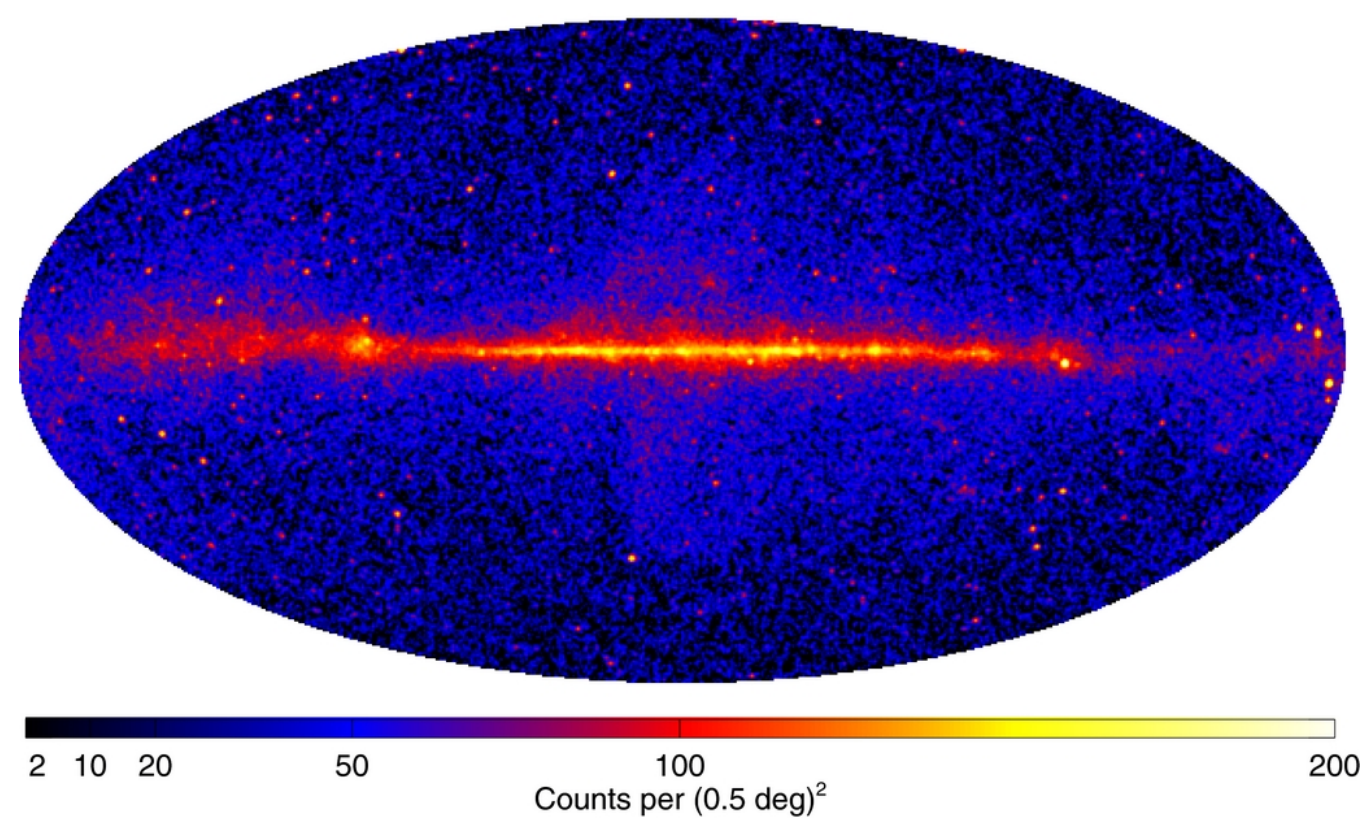

Figure 1: Sky map of gamma-ray counts at $E>10 \mathrm{GeV}$ in Galactic coordinates, from Ackermann et al. (2013)

10) $\times 10^{-11}$ photons $\mathrm{cm}^{-2} \mathrm{~s}^{-1}$ for a source with photon index $\Gamma=2.5$. Out of the 514 sources, $76 \%$ are associated to AGNs, while the fraction of unassociated sources is $13 \%$; the remaining sources are of galactic origin. The fraction of unassociated sources is lower than in the $\mathrm{MeV} / \mathrm{GeV}$ catalogues (where it is around 30\%), mainly thanks to the good source localization achieved at high energy (the $68 \%$ containment radius of the point spread function is $\sim 0^{\circ} .3$ at $10 \mathrm{GeV}$ ).

BL Lac type objects represent the absolute majority of the entire 1FHL (259/514) and among AGNs they outnumber flat spectrum radio quasars (FSRQ, 71 sources), other active galaxies of uncertain type (58), and radio galaxies (5). With the blazar classification based on the SED peak $v_{\mathrm{p}}$, the 1FHL contains 99, 61, 162 low-synchrotron-peaked (LSP, $v_{\mathrm{p}}<10^{14} \mathrm{~Hz}$ ), intermediatesynchrotron-peaked (ISP, $10^{14} \mathrm{~Hz}<v_{\mathrm{p}}<10^{15} \mathrm{~Hz}$ ), and high-synchrotron-peaked (HSP, $v_{\mathrm{p}}>10^{15}$ $\mathrm{Hz}$ ), respectively; for 71 sources it is not possible to provide the SED based classification with the present data.

This distribution is different from the one in the other Fermi catalogues as it leans toward the most extreme spectral type, HSP blazars. These sources are typically fainter in radio; since faint radio sources are generally more difficult to associate with high significance, it is possible that some of them are still among the unassociated share.

\subsection{VLBI observations}

The science goals of our project include securing the blazar classification for many proposed association, shedding light on the nature of the so far unclassified sources, and deriving the physical properties such as the brightness temperature of the confirmed blazars. For all this goals, high angular resolution VLBI observations represent a most valuable resource. After searching the archives for existing observations, we have carried out new observations for the radio sources 


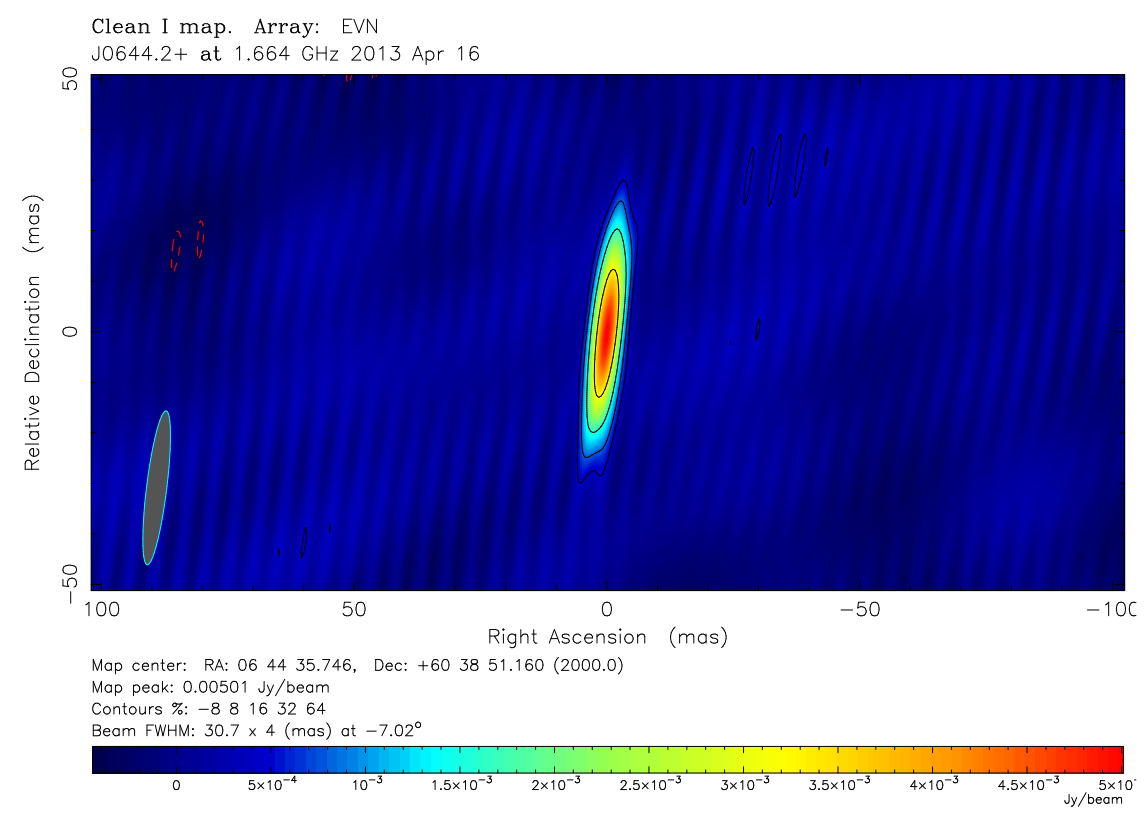

Figure 2: EVN image at $1.6 \mathrm{GHz}$ of the radio counterpart in the field of the unassociated 1FHL source J0644.2+6036. The full width at half maximum beam size is 31 mas $\times 5$ mas in position angle $-7^{\circ}$. The noise level is $0.14 \mathrm{mJy}_{\text {beam }}{ }^{-1}$. The image quality is relatively poor, due to the sparse $(u, v)$-plane coverage and the lack of self-calibration; however, the source is significantly detected.

located in the 1FHL error ellipse without any available data. In particular, we focused on the 269 1FHL sources in the northern hemisphere and we present here the new data obtained for 71 of them with the European VLBI Network. Of these 71 sources, 49 are blazars, 1 is a supernova remnant, and 21 are unassociated.

We carried out the observations at $1.6 \mathrm{GHz}$ in e-VLBI mode during two runs in 2013, one in April (including the long baselines to Shanghai, for sources with declination $\delta>30^{\circ}$ ) and one in June (with baselines to Arecibo, for sources with $0^{\circ}<\delta<30^{\circ}$ ). We observed each target for 18 minutes in phase reference mode, with a dual polarization $512 \mathrm{MHz}$ bandwidth setup. As the coverage of the sky Fourier transform plane is quite sparse, this experiment is most suitable for detection than for proper imaging of the sources. Moreover, most targets are rather weak, with a peak brightness of a few milliJanskys at most, so we could not self-calibrate the data, which further affects the image quality.

\section{Results}

\subsection{VLBI detection rate}

We show in Figure 2 one sample source, selected among those observed in April 2013. The image is quite representative of the overall dataset, with an elongated beam and some remaining calibration issues. However, the significance of the detection is high $(\gg 5 \sigma)$, and a visual inspection of the visibility data confirms the presence of a compact source.

We reveal for the first time compact components in 63 targets, corresponding to a detection rate of $\sim 89 \%$. In particular, we detect radio emission on VLBI scales in every 1FHL source classified 

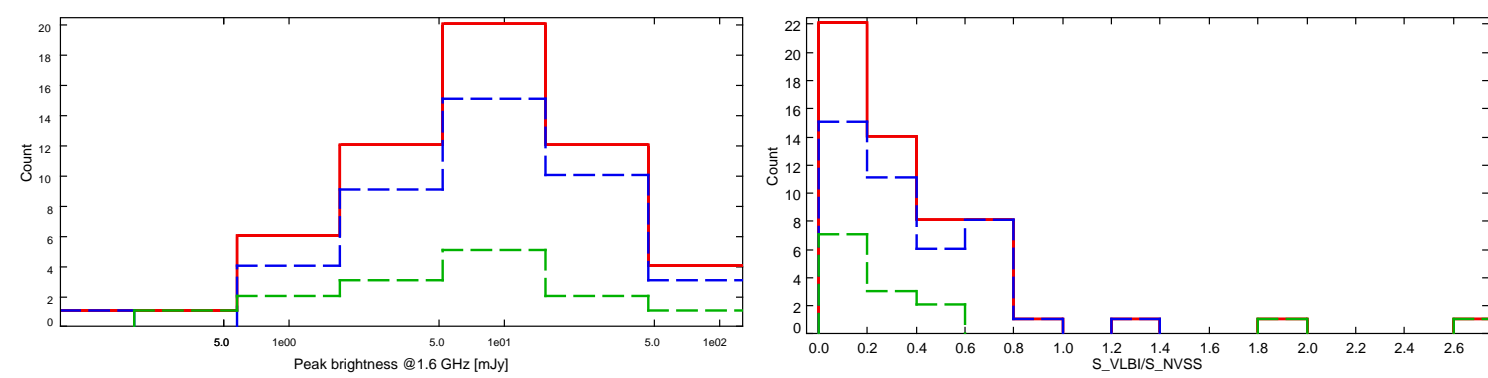

Figure 3: Left: VLBI peak brightness distribution for the EVN observations; right: distribution of the ratio between the VLBI and the NVSS flux density. Solid red line: all sources, blue dashed line: AGNs, green dashed line: unassociated sources.

as blazar. For the unassociated sources, the detection rate is $13 / 21 \sim 62 \%$. We note that several works (D'Abrusco et al., 2013; Massaro et al., 2013; Nori et al., 2014) have proposed a blazar classification for gamma-ray sources based on multi-wavelength properties such as the infrared colours or the low radio frequency spectral index. In the cases of 1FHL sources with one such new proposed associations, we have confirmed the presence of a compact radio source, thus supporting the proposed blazar classification. The source in Figure 2 is indeed one of these cases.

\subsection{Radio and gamma-ray sample properties}

In Figure 3, we show the distribution of the peak brightness in the VLBI data (left panel) and of the ratio between VLBI and NVSS flux density (right panel). In both histograms, we present data based on a preliminary analysis of the new observations, with AGNs and un-associated sources represented by different colours (blue and green, respectively). A full analysis of the entire northern 1FHL sample will be given in a forthcoming paper (Lico et al., in preparation). In general, sources are rather weak, with the VLBI brightness distribution peaking around $\sim 10 \mathrm{mJy}$; moreover, a fair amount of resolved flux is present (peak of $S_{\mathrm{VLBI}} / S_{\mathrm{NVSS}}<0.2$ ). AGNs and unassociated sources are distributed in very similar ways.

In Figure 4, we show the scatter plot of the gamma-ray 1FHL photon flux vs. radio flux density, considering the NVSS (left panel) or the VLBI data (right hand side panel). Again, we indicate AGNs and unassociated sources with blue and green symbols, respectively. The data point are distributed quite uniformly in both plots, suggesting the lack of a strong correlation between radio flux density and $E>10 \mathrm{GeV}$ gamma-ray flux. In any case, the study of the correlation coefficient $r$ reveals that some correlation could be present $(r=0.21)$ in the case of the VLBI vs 1FHL data.

\section{Discussion and future plans}

The preliminary results shown here represent a starting point towards the characterization of the highest energy extragalactic sources. While a detailed analysis will be presented at a later stage, we can already discuss some interesting features based on the results reported here. First, gammaray sources are very frequently associated to the presence of a VLBI compact component. This is not only true for blazar sources, for which it does not come as a surprise, but more interestingly also for unassociated sources. Most likely, this is suggestive that blazars account for most of 

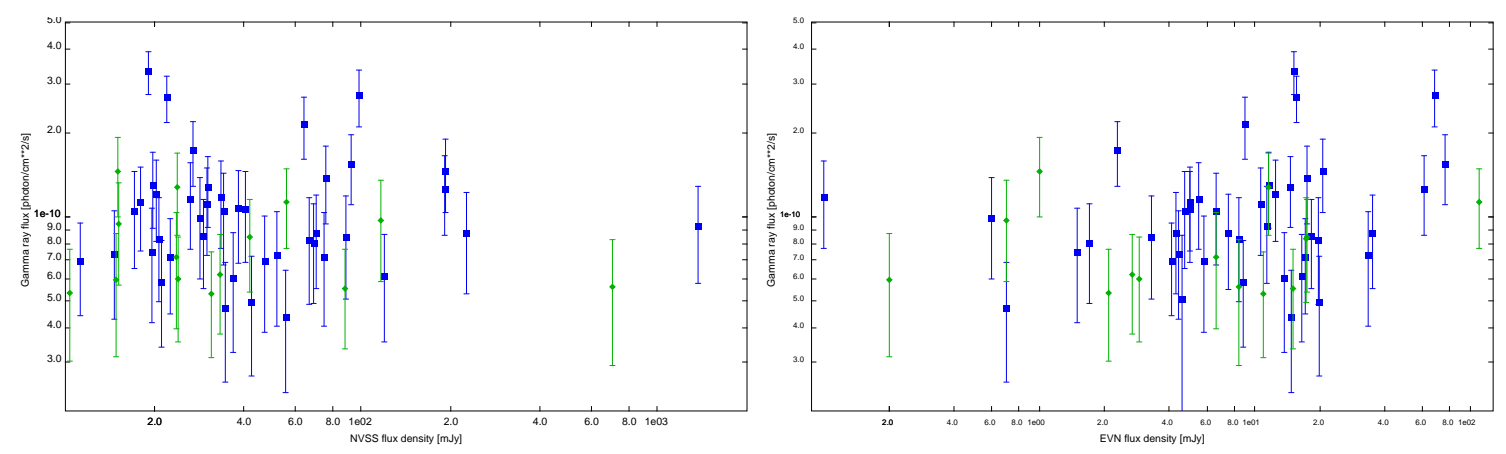

Figure 4: Gamma-ray photon flux vs. radio data preliminary scatter plots, with radio data taken from the NVSS (left panel) or the new EVN observations (right panel). Blue squares represent AGNs, green triangle show un-associated sources.

the hard gamma-ray population. Incidentally, this also lends support to the methods based on complementary multi-wavelength data for the classification of unassociated gamma-ray sources (D’Abrusco et al., 2013; Massaro et al., 2013).

While these results may seem to simply extend the picture already in place for $\mathrm{MeV} / \mathrm{GeV}$ gamma-ray sources, the situation becomes quite different when we consider the detailed radio properties of the observed sources. First, most sources are radio weak. We still do not know if they are weak because they are distant or because they are intrinsically low luminosity sources; either way, typical gamma-ray blazars are at least one magnitude brighter. Moreover, the lack of the radio gamma-ray correlation is quite striking. Ackermann et al. (2011) have revealed a strong and highly significant correlation between radio and gamma rays for the 1LAC blazars, which seems to be missing or at most very weak for the new sample observed here. If this were confirmed in the whole 1FHL, it would indicate that the lack of connection at very high energy does not stem just from an observational bias but rather it has a physical origin. A fully detailed analysis might reveal important details on the blazar sequence (the anti-correlation between synchrotron luminosity and SED peak frequency) or the EBL absorption.

\section{Acknowledgments}

MG acknowledges generous support and hospitality from Curtin Institute for Radio Astronomy and from CSIRO Astronomy and Space Science. MG, RL, FDA, GG, MO acknowledge support from PRIN-INAF-2011.

The European VLBI Network is a joint facility of European, Chinese, South African and other radio astronomy institutes funded by their national research councils. e-VLBI research infrastructure in Europe is supported by the European Union's Seventh Framework Programme (FP7/20072013) under grant agreement number RI-261525 NEXPReS. The research leading to these results has received funding from the European Commission Seventh Framework Programme (FP/20072013) under grant agreement No. 283393 (RadioNet3). 


\section{References}

Abdo, A. A., Ackermann, M., Ajello, M., et al. The First Catalog of Active Galactic Nuclei Detected by the Fermi Large Area Telescope, ApJ,715, 429, [arXiv:1002.0150]

Ackermann, M., Ajello, M., Allafort, A., et al. The Radio/Gamma-Ray Connection in Active Galactic Nuclei in the Era of the Fermi Large Area Telescope, ApJ,741, 30, [arXiv:1108.0501]

Ackermann, M., Ajello, M., Allafort, A., et al. The First Fermi-LAT Catalog of Sources above 10 GeV, ApJS,209, 34

D’Abrusco, R., Massaro, F., Paggi, A., Masetti, N., Tosti, G., Giroletti, M., \& Smith, H. A. Unveiling the Nature of Unidentified Gamma-Ray Sources. I. A New Method for the Association of Gamma-Ray Blazars, ApJS,206, 12, [arXiv:1303.3002]

Fossati, G., Maraschi, L., Celotti, A., Comastri, A., \& Ghisellini, G. A unifying view of the spectral energy distributions of blazars, MNRAS,299, 433, [arXiv:astro-ph/9804103]

Massaro, F., D’Abrusco, R., Giroletti, M., Paggi, A., Masetti, N., Tosti, G., Nori, M., \& Funk, S. Unveiling the Nature of the Unidentified Gamma-Ray Sources. III. Gamma-Ray Blazar-like Counterparts at Low Radio Frequencies, ApJS,207, 4, [arXiv:1308.0330]

Mattox, J. R., Bertsch, D. L., Chiang, J., et al. The Likelihood Analysis of EGRET Data, ApJ,461, 396

Nori, M., Giroletti, M., Massaro, F., D’Abrusco, R., Paggi, A., Tosti, G., \& Funk, S. Unveiling the Nature of Unidentified Gamma-Ray Sources. VI. Gamma-Ray Blazar Candidates in the WISH Survey and their Radio Properties, ApJS,212, 3 